\title{
FIBRE Project: Brazil and Europe Unite Forces and Testbeds for the Internet of the Future
}

\author{
Sebastia Sallent, Antonio Abelém, Iara Machado, Leonardo Bergesio, Serge Fdida, \\ Jose Rezende, Siamak Azodolmolky, Marcos Salvador, Leandro Ciuffo, \\ and Leandros Tassiulas

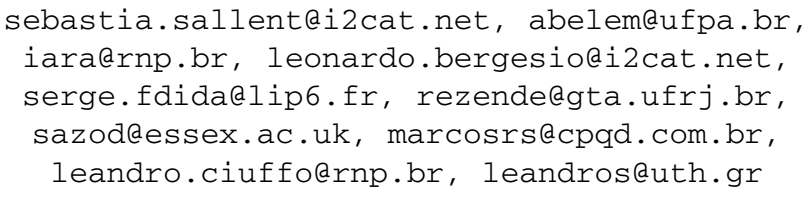

\begin{abstract}
In October 2011 a new, ambitious project was launched, named FIBRE (Future Internet testbeds experimentation between Brazil and Europe). Its main goal is to create common space between Brazil and EU for Future Internet experimental research into network infrastructure and distributed applications, by building and operating a federated EU-Brazil Future Internet experimental facility. Apart from bridging partners from two continents, the project brings together different technologies, including OpenFlow, wireless and optical communications. To demonstrate the public utility of the facility, FIBRE will design and implement a set of pilot applications ranging from seamless wireless connectivity to high-definition content delivery. The poster to be presented describes FIBRE's goals, its testbed facilities and a schema of the envisioned architecture embracing the functionalities of the individual testbeds, as well as their federation. This schema has resulted from the collaborative work of the partners in order to define the functional requirements related to such an architecture. Our ambition with this poster is to attract new users for the facility from the experimental research community, but also to stimulate interest for FIBRE's activities among the conference's attendees.
\end{abstract}

